\title{
Bacterial vaginosis-associated vaginal microbiota is an age-independent risk factor for Chlamydia trachomatis, Mycoplasma genitalium and Trichomonas vaginalis infections in low-risk women, St. Petersburg, Russia
}

\author{
Elena Shipitsyna ${ }^{1}$ (D) $\cdot$ Tatiana Khusnutdinova $^{1,2}$ (D) $\cdot$ Olga Budilovskaya $^{1,2}$ (D) $\cdot$ Anna Krysanova $^{1,2}$ (D) Kira Shalepo $^{1,2}$ (D) \\ Alevtina Savicheva $^{1,2}$ (D) Magnus Unemo $^{3}$ (D)
}

Received: 26 December 2019 / Accepted: 26 January 2020 / Published online: 8 February 2020

(C) The Author(s) 2020

\begin{abstract}
The large majority of studies investigating associations between bacterial vaginosis (BV) and sexually transmitted infections (STIs) have been conducted among predominantly young women with high risk for STIs. Since a risky sexual behavior is a significant risk factor for both STIs and BV, this creates a bias toward an increased association between BV and STIs. This study evaluated associations between BV-associated vaginal microbiota and STIs (Chlamydia trachomatis, Mycoplasma genitalium, Trichomonas vaginalis, and Neisseria gonorrhoeae) in a population of women with low risk for STIs and investigated STI outcomes depending on the dominating Lactobacillus species. Repository cervicovaginal samples collected from reproductive-age women from January 2014 to February 2019 were characterized for vaginal microbiota types and the STIs using multiplex real-time PCR assays. In total, 95 STI-positive and 91 STI-negative samples were included. A significant, age-independent association between BV-associated vaginal microbiota and the presence of $C$. trachomatis, M. genitalium, and T. vaginalis infections was identified (age-adjusted odds ratios 2.92 [95\% confidence interval (CI) 1.24-7.03], 2.88 [95\% CI 1.19-7.16], and $9.75 \times 10^{7}$ [95\% CI 13.03- $\infty$ ], respectively). Normal vaginal microbiota dominated by Lactobacillus crispatus, L. gasseri, or L. jensenii was a strong protective factor against C. trachomatis and/or M. genitalium infections, whereas L. iners-dominated microbiota was not significantly associated with C. trachomatis and/or M. genitalium positivity. The results of the present study confirm that STI prevention strategies should include interventions that also reduce the incidence of BV and promote a protective vaginal microbiota in both high- and low-risk women.
\end{abstract}

Keywords Bacterialvaginosis $\cdot$ Sexually transmitted infections $\cdot$ Chlamydiatrachomatis $\cdot$ Mycoplasmagenitalium $\cdot$ Trichomonas vaginalis $\cdot$ Low-risk women

\section{Introduction}

Sexually transmitted infections (STIs) remain a serious public health problem globally, with Chlamydia

Magnus Unemo

magnus.unemo@regionorebrolan.se

1 Laboratory of Microbiology, D.O. Ott Research Institute of Obstetrics, Gynecology and Reproductology, St. Petersburg, Russia

2 Department of Clinical Laboratory Diagnostics, St. Petersburg State Pediatric Medical University, St. Petersburg, Russia

3 WHO Collaborating Centre for Gonorrhoea and Other STIs, Department of Laboratory Medicine, Faculty of Medicine and Health, SE-701 82, Örebro University, Örebro, Sweden trachomatis, Mycoplasma genitalium, Trichomonas vaginalis, and Neisseria gonorrhoeae infections being the most prevalent non-viral STIs $[1,2]$. In women, STIs can cause infections of lower and upper reproductive tract and result in serious complications and sequelae such as infertility, adverse pregnancy outcomes, and neonatal infections [3-6].

Bacterial vaginosis (BV) is a common vaginal disorder characterized by depletion of the normal Lactobacillus spp.-dominated microbiota and its replacement with an abundance of predominantly anaerobic commensal bacteria, of which Gardnerella vaginalis is considered to play a key role [7]. Clinically, BV is manifested as abnormal malodorous vaginal discharge and associated with significant reproductive morbidity [8]. The prevalence of BV in 
the general population is high worldwide, ranging from 23 to $29 \%$ across regions [9].

There is growing evidence that abnormal vaginal microbiota, primarily that associated with $\mathrm{BV}$, might modulate susceptibility to STIs and be a risk factor for acquisition of STIs. In a recent systematic review and meta-analysis summarizing the data on association between the vaginal microbiota and STIs, a protective role of the vaginal microbiota with high Lactobacillus abundance was shown in relation to $C$. trachomatis, whereas no clear trend for $N$. gonorrhoeae and M. genitalium infections could be detected [10]. The significant association between clinical $\mathrm{BV}$ or $\mathrm{BV}$-associated vaginal microbiota and T. vaginalis infection has been well documented [11-15].

The large majority of the studies that measured the association between $\mathrm{BV}$ (or BV-associated vaginal microbiota) and STIs have been conducted among predominantly young women with a higher risk for STIs, such as STI clinics attendees [16-18], commercial sex workers [19-21], and adolescents or young women with high-risk sexual behavior [22, 23]. Even when studies were conducted among women presenting for routine medical care [12], women recruited from primary care, gynecology, and family planning clinics [24], or rural women from community-based organizations [25], STI positivity rates ranged from relatively high to high. For example, among rural women attending mother's clubs in Peru, who on average had one partner and therefore were considered to be of low risk in regard to STIs, the prevalence of trichomoniasis was $16.5 \%$, C. trachomatis infection $6.8 \%$, gonorrhea $1.2 \%$, and BV (by either Nugent's score or Amsel's criteria) $43.7 \%$ [25]. It is well recognized that BV is associated with risky sexual behavior, and many epidemiological studies support its sexual transmission [26-28], which in populations with high risk and/or high prevalence of STIs creates a bias toward an increased association between BV and STIs.

Vaginal lactobacilli are considered to play a major role in the protection of the female reproductive tract from pathogenic microorganisms as well as BV, with the acidification of the vaginal environment being a main mechanism of the protection [29]. Lactobacillus crispatus, L. iners, L. jensenii, and L. gasseri are the dominating Lactobacillus species [30], but recent studies have suggested that their protective potentials are not equal $[16,18,31]$.

The aims of the present study were to evaluate associations between $\mathrm{BV}$-associated vaginal microbiota, defined using a previously validated multiplex PCR assay [32-34], and STIs (C. trachomatis, M. genitalium, T. vaginalis, and $N$. gonorrhoeae infections) in a population of women at low risk for STIs. In addition, we investigated STI outcomes depending on the vaginal microbiota types, with grouping the normal microbiota into four categories based on the dominating Lactobacillus species.

\section{Materials and methods}

\section{Study design}

This retrospective case-control study was performed at the D.O. Ott Research Institute of Obstetrics, Gynecology and Reproductology (the Ott Institute), St. Petersburg, Russia. Repository cervicovaginal samples from outpatients attending for routine gynecological care, which had been submitted to the Laboratory of Microbiology of the Ott Institute from January 2014 to February 2019 for diagnostic PCR testing for at least one of the non-viral STIs mentioned above, were used in the study. The routine diagnostic testing for $C$. trachomatis, M. genitalium, T. vaginalis, and $N$. gonorrhoeae had been performed using PCR assays developed by InterLabService (Moscow, Russia) and DNA Technology (Moscow, Russia), and all the used PCR assays have been previously validated in comparison with PCR assays commercially available internationally [35-38]. The cervicovaginal swabs had been collected, after removing cervical mucus, from the cervix and vagina with either two separate swabs into the same tube of transport media provided by the manufacturers of the used PCR assays (see above) or, most frequently, the same swab from both sites. The samples after routine testing had been stored at $-70{ }^{\circ} \mathrm{C}$. The samples (STI positive cases and STI negative controls) were selected consecutively in reverse chronological order. Patients' data (age, pregnancy status, and results of STI testing) were obtained from the database of the Laboratory of Microbiology. The inclusion criteria were reproductive age (18-50 years), not being pregnant at the time of sample collection, and not being tested positive for BV and/or any of the four STIs within 2 months prior to enrollment. Sample size was estimated with the use of Fleiss's criterion with continuity correction at the Open Source Epidemiologic Statistics for Public Health (OpenEpi version 3.01) [39] based on assumed proportions of the main exposure (BV-associated vaginal microbiota) of $15 \%$ in controls, $40 \%$ in C. trachomatis and M. genitalium cases, and $90 \%$ in T. vaginalis cases. These proportions were estimated via testing the first 30 STI-negative and 30 STI-positive samples (C. trachomatis $(n=$ 13), M. genitalium ( $n=12)$, and $T$. vaginalis $(n=5)$ ) included in the present study. None of the few $N$. gonorrhoeae samples tested positive during this period was available or eligible. The estimated minimum numbers of positive samples for overall STIs were 57, for $C$. trachomatis and M. genitalium 41 each, and for $T$. vaginalis five samples. The study was approved by the Ethical Committee at the D.O. Ott Research Institute of Obstetrics, Gynecology and Reproductology (approval number 95/2019), and waiver of informed consent was obtained.

\section{DNA isolation}

For the present study, DNA for all PCR assays was isolated from $100 \mu \mathrm{L}$ of sample using the silica-based manual 
extraction kit DNA-Sorb-AM (InterLabService, Moscow, Russia), according to the manufacturer's instructions, with an elution volume of $100 \mu \mathrm{L}$. The DNA preparations were stored at $4{ }^{\circ} \mathrm{C}$ prior to amplification, which was performed within 3 days.

\section{PCR assay for sexually transmitted infections}

Verification of the previously performed routine PCR testing results prior to including the samples in the study was performed using the internationally validated multiplex real-time PCR assay AmpliSens N.gonorrhoeae/C.trachomatis/ M.genitalium/T.vaginalis-MULTIPRIME-FRT (InterLabService, Moscow, Russia) [40].

\section{PCR assays for characterization of the vaginal microbiota}

BV-associated vaginal microbiota was detected using an internationally validated quantitative multiplex real-time PCR assay (AmpliSens Florocenosis/Bacterial vaginosis-FRT; InterLabService, Moscow, Russia), which measures the quantity of G. vaginalis, Atopobium vaginae and Lactobacillus spp. relative to the total bacterial load [32-34]. PCR analysis and interpretation of the results were performed according to the manufacturer's instructions. Ratio coefficients (RC) were calculated as follows: $\mathrm{RC} 1=\lg$ [Lactobacillus spp.] $-\mathrm{lg}$ $[G$. vaginalis $+A$. vaginae $], \mathrm{RC} 2=1 \mathrm{~g}$ [Bacteria $]-\lg$ [Lactobacillus spp.], and $\mathrm{RC} 3=1 \mathrm{~g}$ [Bacteria] $-\mathrm{lg}$ $[G$. vaginalis $+A$. vaginae $]$. A result was interpreted as normal vaginal microbiota if $G$. vaginalis and/or $A$. vaginae were absent or their cumulative concentration was less than the Lactobacillus spp. concentration ( $\mathrm{RC} 1>1)$. A sample was categorized as $\mathrm{BV}$ if $G$. vaginalis and/or A. vaginae concentrations were equal to or exceeded Lactobacillus spp. concentration $(\mathrm{RC} 1<0.5)$. A sample was categorized as intermediate if G. vaginalis and/or A. vaginae concentrations were similar to Lactobacillus spp. concentration $(0.5 \leq \mathrm{RC} 1 \leq 1)$. Finally, a sample was categorized as unspecified microbiota alteration if the concentration of Lactobacillus spp. was decreased, and the concentration of $G$. vaginalis and/or A. vaginae was substantially lower than the concentration of total bacteria $(\mathrm{RC} 2>1$ and $\mathrm{RC} 3>2$ ).

Detection and quantification of L. iners, L. crispatus, $L$. jensenii, and $L$. gasseri was performed using a research kit based on multiplex real-time PCR (DNA Technology, Moscow, Russia), which detects, except for the main four Lactobacillus species, also L. vaginalis, L. acidophilus, and L. johnsonii. Validation of the kit for the present study was performed using clinical isolates of numerous microbial species, including diverse Lactobacillus species, routinely cultured from vaginal samples and species identified using MALDI TOF mass spectrometry analysis in a Microflex instrument (Bruker Daltonics). In the studied cervicovaginal samples with normal microbiota, the dominating Lactobacillus species was determined.

\section{Statistical analyses}

On the basis of PCR characterization of the vaginal microbiota, each sample was assigned to one of the following categories: $\mathrm{BV}$-associated microbiota, intermediate microbiota, unspecified microbiota alteration, $L$. iners-dominated microbiota, $L$. crispatus-dominated microbiota, $L$. jensenii-dominated microbiota, and L. gasseri-dominated microbiota. Distributions of the microbiota types in patients with STIs (combined and individual) were compared with that in STInegative patients using Pearson's chi-square test. Differences in age between patients with STIs (combined and individual) and STI-negative patients were evaluated using the MannWhitney $\mathrm{U}$ test. Univariate logistic regression analysis was used to quantify the strength of association of STIs (combined and individual) with $\mathrm{BV}$-associated microbiota and age and between STIs (combined) and different microbiota types. Multivariate logistic regression was used to estimate ageadjusted odds ratios. Age was included in the logistic regression models in three categories: $18-25,26-35$, and $36-$ 50 years. Tests for significance and confidence intervals in logistic regression were likelihood ratio based. Statistical significance was defined as $p<0.05$ (2-sided) for all analyses. Data were analyzed using JMP 14.3 (SAS Institute, Cary, NC, USA).

\section{Results}

\section{Estimated STI positivity in the target population}

In order to estimate the STI positivity in the target population, data extracted from the database of the Laboratory of Microbiology at the Ott Institute were analyzed. Table 1 summarizes the number and proportion of cervicovaginal samples positive for C. trachomatis, M. genitalium, T. vaginalis, and/ or N. gonorrhoeae from January 2014 to February 2019. C. trachomatis was the most prevalent STI agent (1.4\%), followed by M. genitalium ( $0.5 \%)$, T. vaginalis $(0.3 \%)$, and $N$. gonorrhoeae $(0.2 \%)$.

\section{Selection of cases and controls}

A flowchart describing the selection of cases and controls for the present study is summarized in Fig. 1. In total, 101 STIpositive samples were selected. These included 50 samples positive for $C$. trachomatis, 41 samples positive for M. genitalium, 7 samples positive for $T$. vaginalis, 2 samples positive for C. trachomatis and M. genitalium, and one sample 
Table 1 Number and proportion of cervicovaginal samples positive for Chlamydia trachomatis, Mycoplasma genitalium, Trichomonas vaginalis, and/or Neisseria gonorrhoeae in outpatients attending for routine gynecological care from January 2014 to February 2019

\begin{tabular}{ll} 
Agent & $\begin{array}{l}\text { No. of positive samples/No. } \\
\text { of submitted samples }(\%)\end{array}$ \\
\hline Chlamydia trachomatis & $271 / 19918(1.4)$ \\
Mycoplasma genitalium & $61 / 13021(0.5)$ \\
Trichomonas vaginalis & $12 / 3951(0.3)$ \\
Neisseria gonorrhoeae & $4 / 2598(0.2)$ \\
\hline
\end{tabular}

positive for $C$. trachomatis and T. vaginalis. The included $M$. genitalium and T. vaginalis positive samples constituted all available samples of those tested positive for these infections from January 2014 to February 2019 and meeting the eligibility criteria. The $C$. trachomatis samples represented consecutive samples selected in reverse chronological order in 2019 and 2018. None of the four $N$. gonorrhoeae positive samples were available or eligible. The control group comprised 100 consecutive samples submitted in 2019 for at least one of the STIs and tested negative.

To verify the results of previous routine diagnostic testing and to test for the STIs that were not requested, all selected samples were tested using the AmpliSens N.gonorrhoeae/
C.trachomatis/M.genitalium/T.vaginalis-MULTIPRIME-FRT (InterLabService, Moscow, Russia). All cases were confirmed as positive, but one sample positive for $C$. trachomatis was additionally positive for $M$. genitalium, and one sample positive for $M$. genitalium was additionally positive for $C$. trachomatis (in both cases testing for the second infection had not been performed in the routine diagnostic testing, due to lack of request). All controls were negative for all the tested STIs.

As testing for BV-associated vaginal microbiota was PCR based, total bacterial load $\geq 6 \mathrm{lg}$ genome equivalent (geq)/ml was used, as indicated in the manufacturer's instructions, as a cut-off for sample adequacy. Consequently, 6 case and 9 control samples showing total bacterial load $<6 \mathrm{lg}$ geq $/ \mathrm{ml}$ were excluded. Thus, 95 STI-positive samples (48 samples positive for C. trachomatis, 43 samples positive for M. genitalium, and 8 samples positive for $T$. vaginalis, with 5 cases being mixed infections) and 91 STI-negative samples constituted the final cases and controls in the present study (Fig. 1).

\section{Validation of the multiplex real-time PCR-based research test for the differentiation and quantification of Lactobacillus spp.}

The validation of the Lactobacillus research kit for the present study aimed to confirm that (1) the assay was able to reliably
Fig. 1 Selection of cases and controls. STI, sexually transmitted infection; CT, Chlamydia trachomatis; $\mathrm{MG}$, Mycoplasma genitalium; TV, Trichomonas vaginalis; $\mathrm{NG}$, Neisseria gonorrhoeae; geq, genome equivalent

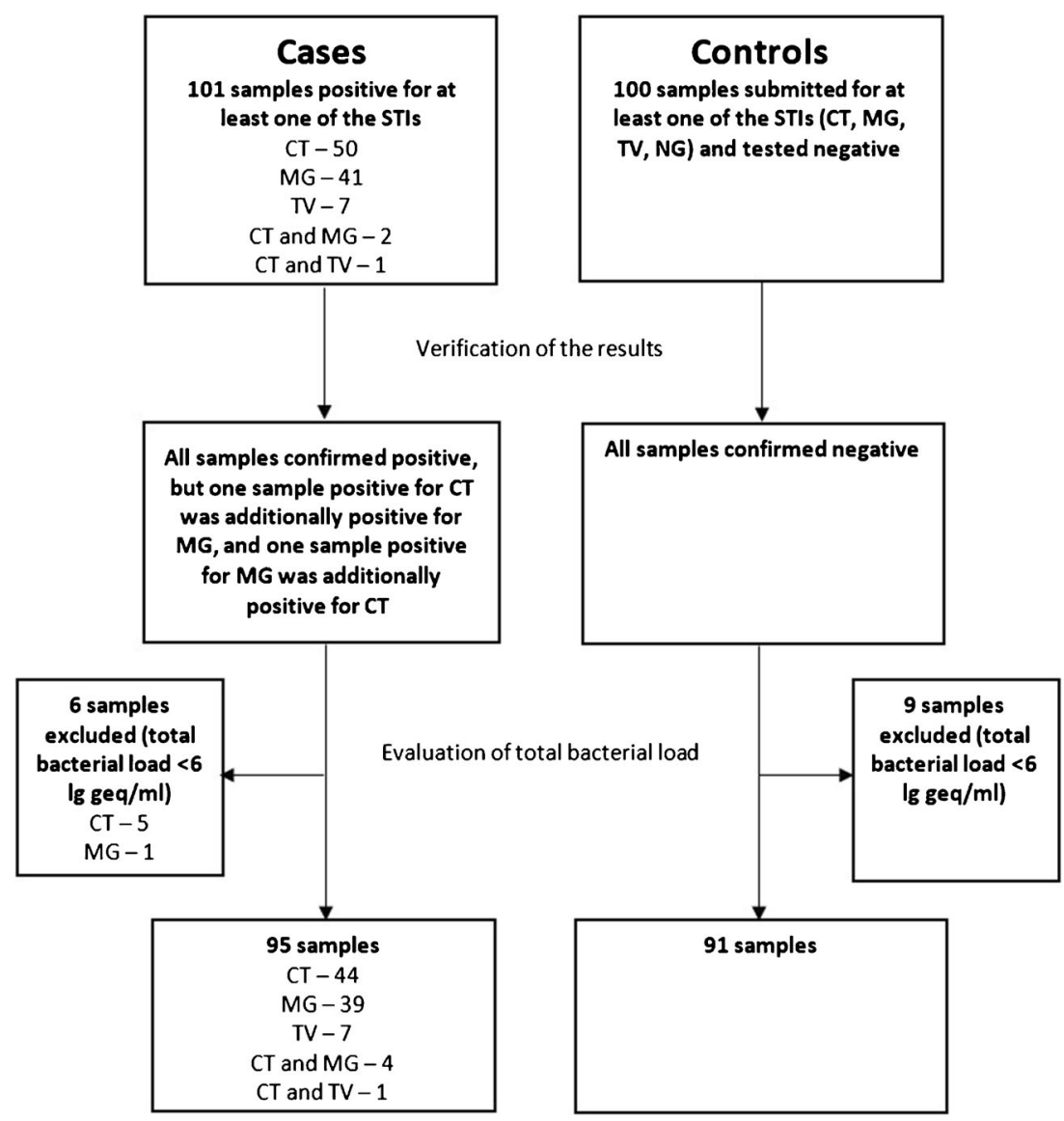


differentiate the four main Lactobacillus species and (2) it did not detect vaginal non-Lactobacillus species. The following clinical vaginal isolates were tested: L. crispatus $(n=9)$, L. iners $(n=4)$, L. jensenii $(n=4)$, L. gasseri $(n=2)$, L. vaginalis $(n=2)$, L. johnsonii $(n=2)$, L. plantarum $(n=$ $2)$, L. rhamnosus $(n=2)$, L. fermentum $(n=1)$, L. paracasei $(n=1)$, Staphylococcus aureus $(n=1)$, Staphylococcus haemolyticus $(n=1)$, Streptococcus agalactiae $(n=1)$, Escherichia coli $(n=1)$, Klebsiella pneumoniae $(n=1)$, Proteus mirabilis $(n=1)$, Enterobacter aerogenes $(n=1)$, Enterobacter cloacea $(n=1)$, Enterococcus faecalis $(n=1)$, Corynebacterium amycolatum $(n=1), G$. vaginalis $(n=1)$, and Candida albicans $(n=1)$. The test correctly detected all targeted Lactobacillus species. None of the non-Lactobacillus isolates tested positive.

\section{Characteristics of women with and without STIs}

A total of 186 women (95 cases and 91 controls) aged 1949 years (median age 31 years, interquartile range $27-$ 35 years) were included. Women with STIs were overall significantly younger (median age 30 years, interquartile range 29-33 years) than women without STIs (median age 32 years, interquartile range 28-37 years; $P=0.0026$ ). This difference mainly reflected the significantly younger age of women with C. trachomatis (median age 27 years, interquartile range 25 34 years; $P=0.0011$ ) and $M$. genitalium (median age 30 years, interquartile range 26-33 years; $P=0.0127$ ), whereas the age of women with $T$. vaginalis did not differ significantly from that of control women (median age 35 years, interquartile range $31-42$ years; $P=0.1611$ ).

Distribution of the vaginal microbiota types in women with STIs differed significantly $(P=0.0022)$ from that in women without STIs (Table 2). The most prominent differences included higher proportions of BV-associated vaginal microbiota in STI-positive women, with all $8 T$. vaginalis samples comprising BV-associated microbiota. Furthermore, samples from STI-positive women contained much lower proportions of vaginal microbiota dominated by L. jensenii and L. gasseri. Notably, in one sample comprising normal microbiota, the only identified Lactobacillus species was L. acidophilus.

\section{Association between sexually transmitted infections, age, and the vaginal microbiota}

Table 3 displays the results of the univariate and multivariate logistic regression models aimed to measure the strength of association of the STIs (combined and individual) with BV-associated microbiota and age. The categories of intermediate microbiota and unspecified microbiota alteration were combined in one category, namely, abnormal non-BV microbiota. Younger age (the categories 19-25 years and, to a lesser degree, 26-35 years) was a strong predictor of both $C$. trachomatis and M. genitalium infections, whereas $T$. vaginalis infection was not significantly associated with age. The odds of detecting STIs, both individual and combined, was significantly higher in women with BVassociated microbiota than in women with normal microbiota (age-adjusted ORs 3.78 [95\% CI 1.84-8.14], 2.92 [95\% CI 1.24-7.03], 2.88 [95\% CI 1.19-7.16], and $9.75 \times 10^{7}$ [95\% CI

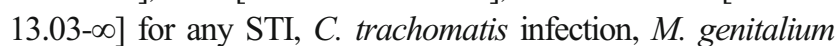
infection, and $T$. vaginalis infection, respectively). Abnormal non-BV microbiota displayed no association with the STIs.

Evaluation of STI outcomes depending on the vaginal microbiota types, with subdivision of the normal microbiota into four categories in accordance with the dominating Lactobacillus species, was performed in a logistic regression analysis combining $C$. trachomatis and $M$. genitalium infections (Table 4). The cases of single $T$. vaginalis infection $(n=7)$ were not included in this analysis because of comprising exclusively BV-associated microbiota. The sample with only $L$. acidophilus was not either included in this analysis. Two approaches were used, one using BV-associated microbiota as reference category and the other using L. crispatus-dominated microbiota as reference category. L. crispatus, L. gasseri, and L. jensenii-dominated microbiota showed strong negative association with $C$. trachomatis and/or M. genitalium infections when compared with BV-associated microbiota, whereas $L$. iners-dominated microbiota was not significantly associated with neither presence nor absence of the STIs. When L. crispatus-dominated microbiota was used as reference category, only BV-associated microbiota was significantly associated with the STIs. Although the odds of detecting STIs in L. iners-dominated microbiota was twice as high as that in L. crispatus-dominated microbiota, the difference did not reach statistical significance. Intermediate microbiota and unspecified microbiota alteration were not significantly associated with the STIs either when compared with BV-associated microbiota or L. crispatus-dominated microbiota.

\section{Discussion}

This study shows that BV-associated vaginal microbiota is an age-independent risk factor for C. trachomatis, M. genitalium, and $T$. vaginalis infections in women with low risk for STIs in St. Petersburg, Russia, with the strongest association demonstrated for $T$. vaginalis infection. The normal vaginal microbiota with domination of L. crispatus, L. jensenii, or L. gasseri had a strong negative association with $C$. trachomatis and/or M. genitalium positivity when compared with BV-associated microbiota, while $L$. iners domination was not associated with presence/absence of these STIs.

This is the first study that provides strong evidence regarding associations between $\mathrm{BV}$ and $C$. trachomatis, M. genitalium, and T. vaginalis infections in women with very low risks for STIs, and it is also one of few studies investigating $\mathrm{BV}$ as a risk factor for $M$. genitalium infection. Although 
Table 2 Vaginal microbiota types in women with and without sexually transmitted infections (STIs)

\begin{tabular}{|c|c|c|c|c|c|}
\hline \multirow[t]{2}{*}{ Vaginal microbiota type } & \multicolumn{5}{|c|}{ No. of samples (\%) } \\
\hline & $\begin{array}{l}\text { STI negative } \\
(n=91)\end{array}$ & Any STI $(n=95)$ & $\begin{array}{l}\text { Chlamydia trachomatis } \\
\text { infection }(n=49)\end{array}$ & $\begin{array}{l}\text { Mycoplasma genitalium } \\
\text { infection }(n=43)\end{array}$ & $\begin{array}{l}\text { Trichom } \\
\text { infection }\end{array}$ \\
\hline \multicolumn{6}{|l|}{ Normal microbiota types } \\
\hline L. iners dominating & $27(29.7)$ & $34(35.8)$ & $17(34.7)$ & $19(44.2)$ & $0(0)$ \\
\hline L. crispatus dominating & $15(16.5)$ & $10(10.5)$ & $8(16.3)$ & $2(4.7)$ & $0(0)$ \\
\hline L. jensenii dominating & $13(14.3)$ & $3(3.2)$ & $1(2.0)$ & $2(4.7)$ & $0(0)$ \\
\hline L. gasseri dominating & $9(9.9)$ & $4(4.2)$ & $2(4.1)$ & $2(4.7)$ & $0(0)$ \\
\hline L. acidophilus dominating & $1(1.1)$ & $0(0)$ & $0(0)$ & $0(0)$ & $0(0)$ \\
\hline \multicolumn{6}{|l|}{ Abnormal microbiota types } \\
\hline BV-associated microbiota & $15(16.5)$ & $38(40.0)$ & $18(36.7)$ & $15(34.9)$ & $8(100)$ \\
\hline Intermediate microbiota & $6(6.6)$ & $3(3.2)$ & $1(2.0)$ & $2(4.7)$ & $0(0)$ \\
\hline Unspecified microbiota alteration & $5(5.5)$ & $3(3.2)$ & $2(4.1)$ & $1(2.3)$ & $0(0)$ \\
\hline$P$ value* & & 0.0022 & 0.0525 & 0.0487 & 0.0002 \\
\hline
\end{tabular}

$P$ values define significance in Pearson's chi-square tests comparing the distributions of the microbiota types in women with STIs (combined and individual) with those in STI-negative women. Significant values are in bold letters. Cases with mixed infections $(n=5)$ were included in all corresponding counts

the examined women represented a subpopulation of outpatients attending the Ott Institute requesting or being offered testing for STIs, the frequency of STI detection was very low, ranging from $0.2 \%$ (N. gonorrhoeae) to $1.4 \%$ (C. trachomatis). Positivity for an STI is a strong epidemiological risk factor for concurrent and subsequent STI [12, 28, 41]. In a previous study [28], incident BV among STI clinic attendees preceded the acquisition of gonorrhea/chlamydia, and gonorrhea/chlamydia appeared to precede the acquisition of BV, which suggests that STIs and BV occur concurrently. In populations with a high risk for STIs, this creates a bias toward increased association between BV and STIs; therefore, we focused on a low-risk population. Our results show a strong association between $\mathrm{BV}$-associated microbiota and infections with $C$. trachomatis, $M$. genitalium, and especially T. vaginalis.

Associations between $\mathrm{BV}$ and $C$. trachomatis and/or $T$. vaginalis have been investigated in many studies; however, only single studies have focused on BV and associations with M. genitalium, with conflicting evidence obtained [20, 22, 42, 43]. In some studies [22, 42], M. genitalium was less frequently detected in women with $\mathrm{BV}$ than in those without $\mathrm{BV}$, whereas BV was an independent risk factor for the infection in other studies [43]. Most recently, prior BV was associated with a 3.5-fold increase in odds of incident $M$. genitalium in a cohort study [20], thereby suggesting that BV may enhance susceptibility to also $M$. genitalium infection. This finding was supported by our results where the odds of detecting $M$. genitalium was nearly 3 times higher in women with BVassociated microbiota than in women with normal microbiota.

The biological, including molecular, mechanisms by which vaginal microbiota affects the risk of STI acquisition remain largely unknown. It is believed that lactic acid and low $\mathrm{pH}$ are the main factors providing protection against STIs. A difference in the protective potential against STIs between different Lactobacillus species has been recently suggested, i.e., particularly L. iners-dominated vaginal microbiota has been associated with an increased $C$. trachomatis risk [16, 18, 31]. These results cannot be directly compared with our findings, because different molecular methods of defining microbiota types were used. Furthermore, since stratification of the cases by the individual infections would have resulted in less statistical power (due to the larger number of microbiota categories), we restricted this analysis to the combination of $C$. trachomatis and $M$. genitalium infections (single $T$. vaginalis infections were excluded because comprised exclusively BV-associated microbiota). The odds of STI detection in samples with L. iners-dominated microbiota was twice as high as that in L. crispatus-dominated microbiota, but twice as low as in those with BV-associated microbiota (non-significant). The dominance of the other three main Lactobacillus species was unambiguously negatively associated with STI presence. Most recently, it was demonstrated that lactic acid plays a major role in the anti-C. trachomatis properties of the vaginal microbiota by modulating host epithelial functions [44]. Moreover, $\mathrm{D}(-)$ lactic acid isomer provided higher protection than $\mathrm{L}(+)$ isomer, which is consistent with the decreased anti$C$. trachomatis properties of $L$. iners that does not produce $\mathrm{D}(-)$ isomer [44]. Accordingly, enhanced understanding of the host response to the cervicovaginal microbiota is crucial, which will contribute to the development of strategies modulating natural protection against STIs.

In this study, analyzing repository samples, the only option for defining $\mathrm{BV}$-associated vaginal microbiota was using a 


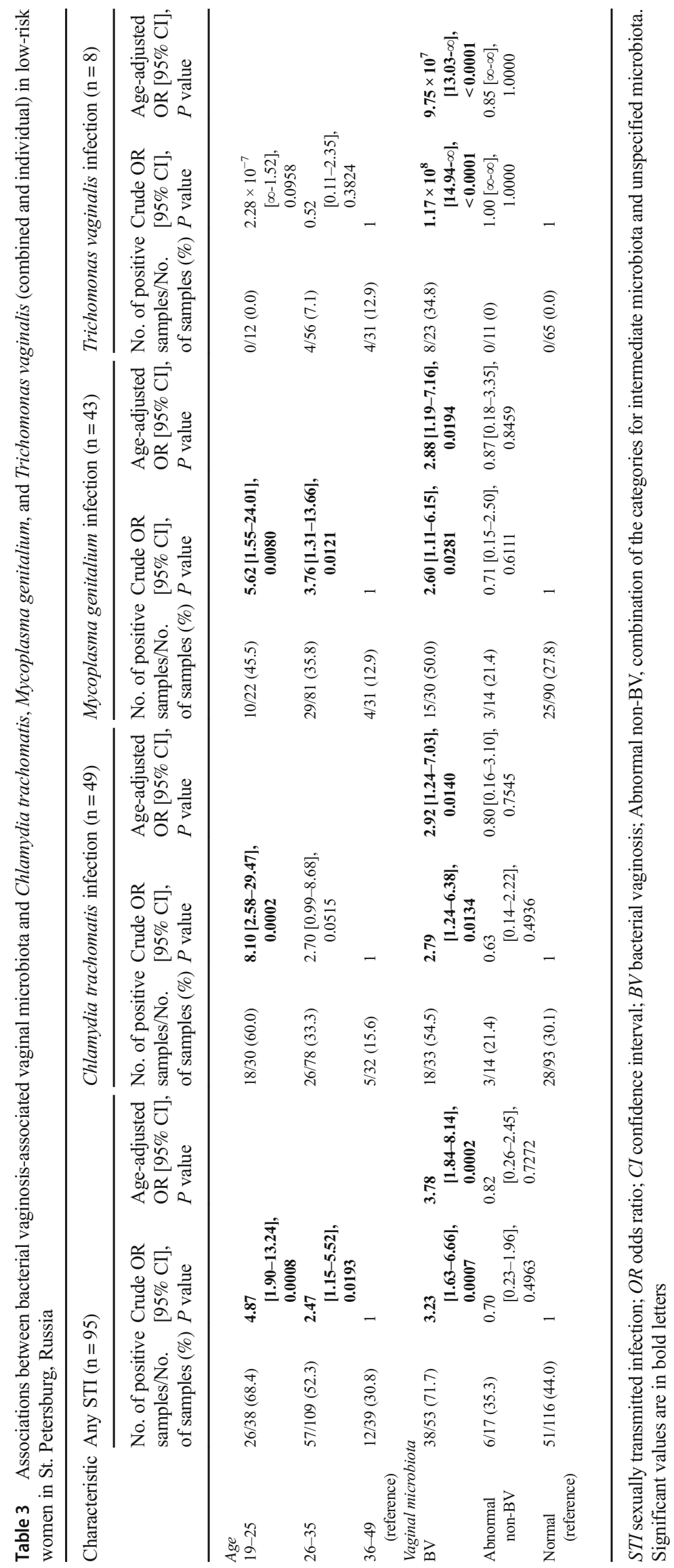


Table 4 Association between vaginal microbiota types and sexually transmitted infections (Chlamydia trachomatis and/or Mycoplasma genitalium) in low-risk women in St. Petersburg, Russia

\begin{tabular}{|c|c|c|c|}
\hline Vaginal microbiota type & $\begin{array}{l}\text { No. of positive } \\
\text { samples/No. of samples (\%) }\end{array}$ & Crude OR $[95 \% \mathrm{CI}], P$ value & Age-adjusted OR $[95 \% \mathrm{CI}], P$ value \\
\hline BV-associated microbiota (reference) & $31 / 46(67.4)$ & 1 & 1 \\
\hline Intermediate microbiota & $3 / 9(33.3)$ & $0.24[0.05-1.10], 0.0578$ & $0.23[0.05-1.16], 0.0756$ \\
\hline Unspecified microbiota alteration & $3 / 8(37.5)$ & $0.29[0.06-1.38], 0.1126$ & $0.39[0.08-2.00], 0.2601$ \\
\hline L. iners dominating & $34 / 61(55.7)$ & $0.61[0.27-1.35], 0.2198$ & $0.56[0.24-1.30], 0.1777$ \\
\hline L. crispatus dominating & $10 / 25(40.0)$ & $0.32[0.12-0.89], 0.0257$ & $0.27[0.09-0.79], 0.0168$ \\
\hline L. jensenii dominating & $3 / 16(18.8)$ & $0.11[0.03-0.45], 0.0006$ & $0.12[0.03-0.49], 0.0035$ \\
\hline L. gasseri dominating & 4/13 (30.8) & $0.22[0.06-0.81], 0.0180$ & $0.20[0.05-0.77], 0.0199$ \\
\hline L. crispatus dominating (reference) & & 1 & 1 \\
\hline BV-associated microbiota & & $3.10[1.13-8.51], 0.0257$ & $3.65[1.26-10.57], 0.0168$ \\
\hline Intermediate microbiota & & $0.75[0.15-3.72], 0.7226$ & $0.84[0.15-4.58], 0.8399$ \\
\hline Unspecified microbiota alteration & & $0.90[0.17-4.64], 0.8996$ & $1.44[0.26-8.07], 0.6816$ \\
\hline L. iners dominating & & 1.89 [0.73-4.87], 0.1840 & $2.06[0.77-5.52], 0.1525$ \\
\hline L. jensenii dominating & & $0.35[0.08-1.53], 0.1447$ & 0.43 [0.09-1.99], 0.2801 \\
\hline L. gasseri dominating & & $0.67[0.16-2.77] .0 .5731$ & $0.71[0.16-3.11], 0.6542$ \\
\hline
\end{tabular}

STI sexually transmitted infection; $O R$ odds ratio; $C I$ confidence interval; $B V$ bacterial vaginosis. Significant values are in bold letters

molecular test. The used test, AmpliSens Florocenosis/ Bacterial vaginosis-FRT, has demonstrated a sensitivity of $93 \%$ and specificity of $86 \%$ when compared to the Nugent score and wet mount microscopy [33], and a sensitivity of 98$100 \%$ and specificity of $91 \%$ compared to the Amsel criteria and $45416 \mathrm{~S}$ rRNA gene pyrosequencing [32]. When compared with the Amsel criteria, Nugent score, culture and another PCR-based assay, BD MAX Vaginal Panel (BD Diagnostics), the AmpliSens Florocenosis/Bacterial vaginosis-FRT test had highest agreement with 16S rRNA gene sequencing [34]. Besides BV-associated vaginal microbiota and normal microbiota, the AmpliSens Florocenosis/ Bacterial vaginosis-FRT test identifies "intermediate microbiota" (G. vaginalis/A. vaginae concentration plus Lactobacillus spp. concentration high) and "unspecified microbiota alteration" ( $G$. vaginalis/A. vaginae concentration plus Lactobacillus spp. concentration low). Our data, based on a low number of samples, suggest that abnormal non-BV microbiota types do not significantly contribute to STI positivity.

The main limitations of our study are associated with the retrospective case-control design, with cross-sectional analysis of samples, which makes it impossible to determine causality. A longitudinal cohort study with sufficiently frequent testing for BV, enabling to detect BV prior to STI acquisition, would make it possible to establish causal relation between BV and STIs; however, it will be exceedingly challenging to achieve sufficient sample size to observe STI outcomes in a population with low STI positivity. A molecular test for the evaluation of the vaginal microbiota could enable to avoid prospective testing of all patients at every visit for BV- associated microbiota and to restrict the analysis to the samples obtained immediately prior to STI acquisition. Ideally, the molecular method should both differentiate and quantify the bacterial community state types and accurately define BVassociated microbiota. Furthermore, relevant patient data were lacking, e.g., regarding recent antimicrobial use. To limit this bias, we excluded women who had any urogenital samples positive for the four STIs and/or BV within 2 months prior to enrollment, and any antimicrobial use did likely not differ significantly in STI-positive versus STI-negative patients. We were additionally lacking information on sexual activity, STI history, and other potential confounders. However, controlling for age, one of the strongest confounders, did not significantly change the association between BV-associated microbiota and the STIs. Furthermore, we used samples submitted for STI testing rather than for vaginal microbiota evaluation. According to our clinical practice, the major specimen for C. trachomatis and M. genitalium detection in women is cervicovaginal sample. For T. vaginalis detection, vaginal swabs are mostly used. Nevertheless, it has been shown that the microbiota in the cervix and the vagina is generally similar $[16,45]$. The cervical microbiota might be less abundant in bacteria than the vaginal microbiota, but we assessed relative rather than absolute bacterial loads, and we did not include samples with low bacterial load $(<6 \mathrm{lg} \mathrm{geq} / \mathrm{ml})$; therefore, it appears unlikely that the difference in the bacterial abundance between the cervix and vagina has largely affected the results. Finally, due to the few $N$. gonorrhoeae positive samples, no associations between $\mathrm{BV}$ and gonorrhea could be examined. In a recent meta-analysis, measures of association between $\mathrm{BV}$ and gonorrhea in the 8 included studies ranged from 
0.80 to 3.75 , with only one study showing a significant association [10]. Consequently, more data is imperative.

In conclusion, we show a significant, age-independent association between BV-associated microbiota and C. trachomatis, M. genitalium, and T. vaginalis infections also in women with low risk for STIs. Our findings suggest that the normal vaginal microbiota dominated by $L$. crispatus, $L$. gasseri, and $L$. jensenii is a strong protective factor against STIs, while L. iners-dominated microbiota is not significantly associated with STI outcomes. Our results confirm that STI prevention strategies should include interventions that reduce the incidence of $\mathrm{BV}$ and promote a protective vaginal microbiota.

Funding Information Open access funding provided by Örebro University. The study was supported by the Ministry of Science and Higher Education of the Russian Federation (contract no. AAAA-A19119-021290030-0). The research kits for Lactobacillus detection and differentiation were unconditionally granted by the manufacturer (DNA Technology, Moscow, Russia). The funders had no role in study design, data collection and analysis, decision to publish, or preparation of the manuscript.

\section{Compliance with ethical standards}

Conflict of interest The authors declare that they have no conflicts of interest.

Open Access This article is licensed under a Creative Commons Attribution 4.0 International License, which permits use, sharing, adaptation, distribution and reproduction in any medium or format, as long as you give appropriate credit to the original author(s) and the source, provide a link to the Creative Commons licence, and indicate if changes were made. The images or other third party material in this article are included in the article's Creative Commons licence, unless indicated otherwise in a credit line to the material. If material is not included in the article's Creative Commons licence and your intended use is not permitted by statutory regulation or exceeds the permitted use, you will need to obtain permission directly from the copyright holder. To view a copy of this licence, visit http://creativecommons.org/licenses/by/4.0/.

\section{References}

1. Rowley J, Vander Hoorn S, Korenromp E, Low N, Unemo M, AbuRaddad LJ et al (2019) Chlamydia, gonorrhoea, trichomoniasis and syphilis: global prevalence and incidence estimates, 2016. Bull World Health Organ 97:548-562. https://doi.org/10.2471/blt.18.228486

2. Baumann L, Cina M, Egli-Gany D, Goutaki M, Halbeisen FS, Lohrer GR et al (2018) Prevalence of Mycoplasma genitalium in different population groups: systematic review andmeta-analysis. Sex Transm Infect 94:255-261. https://doi.org/10.1136/sextrans2017-053384

3. Wiesenfeld HC, Manhart LE (2017) Mycoplasma genitalium in women: current knowledge and research priorities for this recently emerged pathogen. J Infect Dis 216:S389-S395. https://doi.org/10. 1093/infdis/jix198

4. Blas MM, Canchihuaman FA, Alva IE, Hawes SE (2007) Pregnancy outcomes in women infected with Chlamydia trachomatis: a population-based cohort study in Washington state. Sex Transm Infect 83:314-318. https://doi.org/10.1136/sti.2006. 022665

5. Silver BJ, Guy RJ, Kaldor JM, Jamil MS, Rumbold AR (2014) Trichomonas vaginalis as a cause of perinatal morbidity: a systematic review and meta-analysis. Sex Transm Dis 41:369-376. https:// doi.org/10.1097/OLQ.0000000000000134

6. Lochner HJ, Maraqa NF (2018) Sexually transmitted infections in pregnant women: integrating screening and treatment into prenatal care. Paediatr Drugs 20:501-509. https://doi.org/10.1007/s40272018-0310-4

7. Muzny CA, Taylor CM, Swords WE, Tamhane A, Chattopadhyay $\mathrm{D}$, Cerca $\mathrm{N}$ et al (2019) An updated conceptual model on the pathogenesis of bacterial vaginosis. J Infect Dis 220:1399-1405. https:// doi.org/10.1093/infdis/jiz342

8. Kroon SJ, Ravel J, Huston WM (2018) Cervicovaginal microbiota, women's health, and reproductive outcomes. Fertil Steril 110:327336. https://doi.org/10.1016/j.fertnstert.2018.06.036

9. Peebles K, Velloza J, Balkus JE, McClelland RS, Barnabas RV (2019) High global burden and costs of bacterial vaginosis: a systematic review and meta-analysis. Sex Transm Dis 46:304-311. https://doi.org/10.1097/OLQ.0000000000000972

10. Tamarelle J, Thiébaut ACM, de Barbeyrac B, Bébéar C, Ravel J, Delarocque-Astagneau E (2019) The vaginal microbiota and its association with human papillomavirus, Chlamydia trachomatis, Neisseria gonorrhoeae and Mycoplasma genitalium infections: a systematic review and meta-analysis. Clin Microbiol Infect 25: 35-47. https://doi.org/10.1016/j.cmi.2018.04.019

11. Brotman RM, Bradford LL, Conrad M, Gajer P, Ault K, Peralta L et al (2012) Association between Trichomonas vaginalis and vaginal bacterial community composition among reproductive-age women. Sex Transm Dis 39:807-812. https://doi.org/10.1097/ OLQ.0b013e3182631c79

12. Brotman RM, Klebanoff MA, Nansel TR, Yu KF, Andrews WW, Zhang J et al (2010) Bacterial vaginosis assessed by gram stain and diminished colonization resistance to incident gonococcal, chlamydial, and trichomonal genital infection. J Infect Dis 202:19071915. https://doi.org/10.1086/657320

13. Jarrett O, Srinivasan S, Richardson B, Fiedler T, Wallis J, Kinuthia J et al (2019) Specific vaginal bacteria are associated with increased risk of Trichomonas vaginalis acquisition in women. J Infect Dis 220:1503-1510. https://doi.org/10.1093/infdis/jiz354

14. Balkus J, Richardson B, Rabe L, Taha T, Mgodi N, Phiri Kasaro M et al (2014) Bacterial vaginosis and the risk of Trichomonas vaginalis acquisition among HIV-1 negative women. Sex Transm Dis 41:123-128. https://doi.org/10.1097/OLQ.0000000000000075

15. Abbai NS, Reddy T, Ramjee G (2016) Prevalent bacterial vaginosis infection - a risk factor for incident sexually transmitted infections in women in Durban, South Africa. Int J STD AIDS 27:1283-1288. https://doi.org/10.1177/0956462415616038

16. Van Der Veer C, Bruisten SM, Van Der Helm JJ, De Vries HJC, Van Houdt R (2017) The cervicovaginal microbiota in women notified for Chlamydia trachomatis infection: a case-control study at the sexually transmitted infection outpatient clinic in Amsterdam, the Netherlands. Clin Infect Dis 64:24-31. https://doi.org/10.1093/cid/ ciw586

17. Wiesenfeld HC, Hillier SL, Krohn MA, Landers DV, Sweet RL (2003) Bacterial vaginosis is a strong predictor of Neisseria gonorrhoeae and Chlamydia trachomatis infection. Clin Infect Dis 36:663-668. https://doi.org/10.1086/367658

18. Tamarelle J, De Barbeyrac B, Le Hen I, Thiébaut A, Bébéar C, Ravel J et al (2018) Vaginal microbiota composition and association with prevalent Chlamydia trachomatis infection: a cross-sectional study of young women attending a STI clinic in France. Sex Transm Infect 94:616-618. https://doi.org/10.1136/sextrans-2017-053346 
19. Kaul R, Nagelkerke N, Kimani J, Ngugi E, Bwayo JJ, MacDonald $\mathrm{KS}$ et al (2007) Prevalent herpes simplex virus type 2 infection is associated with altered vaginal flora and an increased susceptibility to multiple sexually transmitted infections. J Infect Dis 196:16921697. https://doi.org/10.1086/522006

20. Lokken EM, Balkus JE, Kiarie J, Hughes JP, Jaoko W, Totten PA et al (2017) Association of recent bacterial vaginosis with acquisition of Mycoplasma genitalium. Am J Epidemiol 186:194-201. https://doi.org/10.1093/aje/kwx043

21. Martin HL, Richardson BA, Nyange PM, Lavreys L, Hillier SL, Chohan B et al (1999) Vaginal lactobacilli, microbial flora, and risk of human immunodeficiency virus type 1 and sexually transmitted disease acquisition. J Infect Dis 180:1863-1868. https://doi.org/10. $1086 / 315127$

22. Huppert J, Bates J, Weber A, Quinn N, Gaydos C (2013) Abnormal vaginal $\mathrm{pH}$ and Mycoplasma genitalium infection. J Pediatr Adolesc Gynecol 26:36-39. https://doi.org/10.1016/j.jpag.2012. 09.005

23. Allsworth J, Peipert J (2011) Severity of bacterial vaginosis and the risk of sexually transmitted infection. Am J Obstet Gynecol 205: 113.e1-113.e6. https://doi.org/10.1016/j.ajog.2011.02.060

24. Peipert JF, Lapane KL, Allsworth JE, Redding CA, Blume JD, Stein MD (2008) Bacterial vaginosis, race, and sexually transmitted infections: does race modify the association? Sex Transm Dis 35: 363-367. https://doi.org/10.1097/OLQ.0b013e31815e4179

25. García PJ, Chavez S, Feringa B, Chiappe M, Li W, Jansen KU et al (2004) Reproductive tract infections in rural women from the highlands, jungle, and coastal regions of Peru. Bull World Health Organ $82: 483-492$

26. Bradshaw CS, Walker J, Fairley CK, Chen MY, Tabrizi SN, Donovan B et al (2013) Prevalent and incident bacterial vaginosis are associated with sexual and contraceptive behaviours in young Australian women. PLoS One 8:1-8. https://doi.org/10.1371/ journal.pone. 0057688

27. Vodstrcil LA, Walker SM, Hocking JS, Law M, Forcey DS, Fehler $\mathrm{G}$ et al (2015) Incident bacterial Vaginosis (BV) in women who have sex with women is associated with behaviors that suggest sexual transmission of BV. Clin Infect Dis 60:1042-1053. https:// doi.org/10.1093/cid/ciu1130

28. Gallo MF, Macaluso M, Warner L, Fleenor ME, Hook EW, Brill I et al (2012) Bacterial vaginosis, gonorrhea, and chlamydial infection among women attending a sexually transmitted disease clinic: a longitudinal analysis of possible causal links. Ann Epidemiol 22: 213-220. https://doi.org/10.1016/j.annepidem.2011.11.005

29. Graver MA, Wade JJ (2011) The role of acidification in the inhibition of Neisseria gonorrhoeae by vaginal lactobacilli during anaerobic growth. Ann Clin Microbiol Antimicrob 10:1-5. https://doi. org/10.1186/1476-0711-10-8

30. Ravel J, Gajer P, Abdo Z, Schneider GM, Koenig SSK, McCulle SL et al (2011) Vaginal microbiome of reproductive-age women. Proc Natl Acad Sci U S A 108:4680-4687. https://doi.org/10.1073/pnas. 1002611107

31. van Houdt R, Ma B, Bruisten S, Speksnijder A, Ravel J, de Vries H (2018) Lactobacillus iners-dominated vaginal microbiota is associated with increased susceptibility to Chlamydia trachomatis infection in Dutch women, a case control study. Sex Transm Infect 94: 117-123. https://doi.org/10.1109/EMBC.2016.7590696

32. Rumyantseva T, Shipitsyna E, Guschin A, Unemo M (2016) Evaluation and subsequent optimizations of the quantitative AmpliSens Florocenosis/bacterial vaginosis-FRT multiplex realtime PCR assay for diagnosis of bacterial vaginosis. APMIS 124: 1099-1108. https://doi.org/10.1111/apm.12608

33. Rumyantseva TA, Bellen G, Romanuk TN, Shipulina OI, Guschin AE, Shipulin GA et al (2015) Utility of microscopic techniques and quantitative real-time polymerase chain reaction for the diagnosis of vaginal microflora alterations. J Low Genit Tract Dis 19:124-128. https://doi.org/10.1097/LGT.0000000000000060

34. van den Munckhof EHA, van Sitter RL, Boers KE, Lamont RF, te Witt R, le Cessie S et al (2019) Comparison of Amsel criteria, Nugent score, culture and two CE-IVD marked quantitative realtime PCRs with microbiota analysis for the diagnosis of bacterial vaginosis. Eur J Clin Microbiol Infect Dis 38:959-966. https://doi. org/10.1007/s10096-019-03538-7

35. Shipitsyna E, Zolotoverkhaya E, Chen CY, Chi KH, Grigoryev A, Savicheva A et al (2013) Evaluation of polymerase chain reaction assays for the diagnosis of Trichomonas vaginalis infection in Russia. J Eur Acad Dermatol Venereol 27:e217-e223. https://doi. org/10.1111/j.1468-3083.2012.04593.x

36. Shipitsyna E, Zolotoverkhaya E, Krysanova A, Savicheva A, Sokolovsky E, Domeika M et al (2009) First evaluation of six nucleic acid amplification tests widely used in the diagnosis of Chlamydia trachomatis in Russia. J Eur Acad Dermatol Venereol 23:268-276. https://doi.org/10.1111/j.1468-3083.2008.03038.x

37. Shipitsyna E, Zolotoverkhaya E, Hjelmevoll SO, Maximova A, Savicheva A, Sokolovsky E et al (2009) Evaluation of six nucleic acid amplification tests used for diagnosis of Neisseria gonorrhoeae in Russia compared with an international strictly validated real-time porA pseudogene polymerase chain reaction. J Eur Acad Dermatol Venereol 23:1246-1253. https://doi.org/10.1111/j. 1468-3083.2009.03290.x

38. Shipitsyna E, Zolotoverkhaya E, Dohn B, Benkovich A, Savicheva A, Sokolovsky E et al (2009) First evaluation of polymerase chain reaction assays used for diagnosis of Mycoplasma genitalium in Russia. J Eur Acad Dermatol Venereol 23:1164-1172. https://doi. org/10.1111/j.1468-3083.2009.03276.x

39. Sullivan KM, Dean A, Soe MM (2017) On academics : OpenEpi: a web-based epidemiologic and statistical calculator for public health. Public Health Rep 124:471-474. https://doi.org/10.1177/ 003335490912400320

40. Rumyantseva T, Golparian D, Nilsson CS, Johansson E, Falk M, Fredlund $\mathrm{H}$ et al (2015) Evaluation of the new AmpliSens multiplex real-time PCR assay for simultaneous detection of Neisseria gonorrhoeae, Chlamydia trachomatis, Mycoplasma genitalium, and Trichomonas vaginalis. APMIS 123:879-886. https://doi.org/ 10.1111/apm. 12430

41. Kenyon C, Buyze J, Klebanoff M, Brotman RM (2018) The role of sexual networks in studies of how BV and STIs increase the risk of subsequent reinfection. Epidemiol Infect 146:2003-2009. https:// doi.org/10.1017/S0950268818002157

42. Manhart LE, Critchlow CW, Holmes KK, Dutro SM, Eschenbach DA, Stevens CE et al (2003) Mucopurulent cervicitis and Mycoplasma genitalium. J Infect Dis 187:650-657. https://doi. org/10.1086/367992

43. Oakeshott P, Aghaizu A, Hay P, Reid F, Kerry S, Atherton H et al (2010) Is Mycoplasma genitalium in women the "new chlamydia?" a community-based prospective cohort study. Clin Infect Dis 51: 1160-1166. https://doi.org/10.1086/656739

44. Edwards V, Smith S, McComb E, Tamarelle J, Ma B, Humphrys M et al (2019) The cervicovaginal microbiota-host interaction modulates Chlamydia trachomatis infection. MBio 10:e01548-e1519. https://oi.org/10.1128/mbio.01548-19

45. Smith WL, Hedges SR, Mordechai E, Adelson ME, Trama JP, Gygax SE et al (2014) Cervical and vaginal flora specimens are highly concordant with respect to bacterial vaginosis-associated organisms and commensal Lactobacillus species in women of reproductive age. J Clin Microbiol 52:3078-3081. https://doi.org/10. 1128/JCM.00795-14

Publisher's note Springer Nature remains neutral with regard to jurisdictional claims in published maps and institutional affiliations. 